\title{
Kinetics of CD11b/CD18 Up-Regulation During Infection with the Agent of Human Granulocytic Ehrlichiosis in Mice
}

\author{
Dori L. Borjesson, Scott I. Simon, Emir Hodzic, Christie M. Ballantyne, and \\ Stephen W. Barthold
}

Center for Comparative Medicine, Schools of Medicine and Veterinary Medicine (DLB, EH, SWB) and Biomedical Engineering (SIS), University of California, Davis, California; and Section of Atherosclerosis and Leukocyte Biology $(\mathrm{CMB})$, Departments of Medicine and Pediatrics, Baylor College of Medicine, Houston, Texas

\begin{abstract}
SUMIMARY: The agent of human granulocytic ehrlichiosis (aoHGE) is a tick-borne, obligate intracellular, granulocytotropic bacterium able to infect numerous host species. Given its unique niche and the leukopenia often noted with infection, we investigated the effect of acute aoHGE infection on neutrophil activation by evaluating surface expression of the $\beta 2$ integrin $\mathrm{CD} 11 \mathrm{~b} / \mathrm{CD} 18$ in a mouse model using FACS analysis. Infection resulted in neutrophil activation with up-regulation of CD11b/CD18 in multiple strains of mice, however, hematologic analysis showed no apparent role for CD11b/CD18 in mediating peripheral leukopenia. Because IFN- $\gamma$ is an important cytokine during granulocytic ehrlichiosis and is known to activate leukocytes, we investigated the potential role of IFN- $\gamma$ in CD11b/CD18 up-regulation. Neutrophils from IFN- $\gamma$ knock-out mice became activated during aoHGE infection, however, the kinetics of activation differed from wild-type mice. In addition, activation correlated directly with the presence of bacteria because neutrophils with large intracytoplasmic morula also expressed higher levels of CD11b/CD18. CD11b/CD18 seemed to be critical to early bacterial clearance and killing in vivo because infection of mice with targeted genetic disruption of CD11b/CD18 resulted in an initial increase in bacterial burden compared with wild-type mice. Similarly, in vitro culture of neutrophils from infected CD11b/CD18 knock-out mice resulted in a marked increase in bacterial proliferation compared with congenic controls. The data support crucial roles of CD11b/CD18 and IFN- $\gamma$-mediated cell activation as mechanisms that limit bacterial replication. (Lab Invest 2002, 82:303-311).
\end{abstract}

$H$ uman granulocytic ehrlichiosis (HGE) is a tickborne, zoonotic disease caused by an obligate intracellular granulocytotropic bacterium (Chen et al, 1994). Ehrlichia species infect a wide array of mammalian hosts. Although long recognized as important veterinary pathogens, Ehrlichia species have only recently emerged as significant human pathogens (Anderson et al, 1991; Chen et al, 1994; Walker and Dumler, 1996). The majority of Ehrlichia species selectively inhabit unique, intracellular niches within granulocytes, monocytes, platelets, or erythrocytes, yet clinical signs are remarkably similar across both Ehrlichia and host species.

Laboratory mice serve as useful tools for investigation of HGE pathogenesis and infection kinetics. Immunocompetent $\mathrm{C} 3 \mathrm{H} / \mathrm{HeN}$ mice recover from infection, but $\mathrm{C} 3 \mathrm{H}-\mathrm{SCID}$ mice remain persistently infected.

Received November 8, 2001

This work was supported by Grants AI-41440 and RR-07038 (to SWB), AI-47294 (to SIS), and R01-HL62243-01 (to CMB) from the National Institutes of Health. SIS and CMB are Established Investigators of the American Heart Association. DLB is supported by NIH Training Grant T32 RR-07038.

Address reprint requests to: Dr. Stephen W. Barthold, University of California-Davis, Center for Comparative Medicine, One Shields Ave, Davis, CA 95616. E-mail: swbarthold@ucdavis.edu
In contrast, C57BL/6 mice show different infection kinetics and support a transient and low infection burden (Hodzic et al, 1998). Combining the mouse model with quantitative PCR of blood and other tissues permits investigation of infection kinetics not accessible with in vitro or cell culture systems. Additionally, the use of genetically engineered mice with precise genetic defects, such as adhesion molecule and cytokine knock-out mice, allows detailed investigation of the correlation between infection kinetics, pathogenesis, and leukocyte activation.

Because of the unique reliance of the agent of human granulocytic ehrlichiosis (aoHGE) on a host granulocyte, neutrophil adhesion molecules may play a variety of roles during host infection including dissemination within the host and transmission to a competent vector. As such, intracellular infection with the aoHGE may result in alterations in neutrophil adhesive or chemotactic receptors. Specifically in response to inflammatory stimuli, neutrophils undergo a multistep process of emigration initiated by cell rolling along endothelial cells, which is followed by firm adhesion and transmigration into tissues. Initial tethering to endothelium is mediated by the selectin family of adhesion molecules, whereas firm adhesion and transmigration are dependent on activated $\beta 2$ integrins, including CD11a/CD18 (leukocyte function 
associated antigen-1) and CD11b/CD18 (Mac-1, CR3) (Dib, 2000). Activation of CD11b/CD18 is a prerequisite for several proinflammatory functions including the oxidative burst, an increase in intracellular calcium, and the induction of gene expression. In addition to aiding firm endothelial adhesion, CD11b/CD18 is an important receptor for attachment to opsonized bacteria (primary ligand for iC3b), it binds mannose and other carbohydrates, and it mediates homotypic neutrophil aggregation (Berton et al, 1996; Dib, 2000; Lu et al, 1997; Simon et al, 1992).

Although both $\beta 2$ integrins are constitutively expressed on the neutrophil surface, only CD11b/CD18 is up-regulated from readily mobilizable cytoplasmic storage pools upon activation through a variety of cell surface receptors. IFN- $\gamma$ is a broadly acting cytokine whose actions include up-regulation of the respiratory burst, neutrophil and monocyte activation, and degranulation among many others (Aas et al, 1999; Gaviria et al, 1999; Kullberg et al, 1993). As a T helper 1 cytokine, IFN- $\gamma$ is thought to play a primary role in host defenses against intracellular microbes. Indeed, IFN- $\gamma$ has been shown to dominate the murine cytokine response to aoHGE infection and limit early bacteremia (Akkoyunlu and Fikrig, 2000; Martin et al, 2001). However the role of IFN- $\gamma$ in neutrophil activation during aoHGE infection is unknown.

In the present study, we investigated the effect of acute aoHGE infection on neutrophil activation in mice. We hypothesized that acute aoHGE infection would result in alterations of CD11b/CD18 expression on the neutrophil surface, which may be important in peripheral leukopenia, bacterial survival, and cell clearance. We found that during acute infection, CD11b/CD18 up-regulation was a consistent feature of disease, was directly associated with the presence of bacteria, and occurred in the absence of IFN- $\gamma$ production. However IFN- $\gamma$ was important in controlling bacterial proliferation and clearance over the course of infection. In addition, the absence of CD11b/ CD18 promoted an early increase in infection burden. CD11b/CD18 may be linked to intracellular killing and clearance of infection because aoHGE replicated more effectively in CD11b (-/-) neutrophils cultured ex vivo. Finally, CD11b/CD18 did not seem to play a role in the leukopenia.

\section{Results}

\section{Kinetics of Leukopenia in Mice Strains}

Leukocyte numbers in blood decreased in $\mathrm{C} 3 \mathrm{H}, \mathrm{B} 6$, and B6-CD11b (-/-) mice as depicted in Figure 1. Leukopenia was characterized by a neutropenia and moderate lymphopenia. Because uninfected mice from all strains did not show significant differences in leukocyte number at any time point, they were combined for analysis. Infected animals were significantly more leukopenic than uninfected controls on Days 3 and 4 after infection $(p=0.0004$ and $p=0.003$, respectively). Although the kinetics of leukopenia varied somewhat between strains, because of high indi-



Figure 1.

Mean leukocyte counts in aoHGE (agent of human granulocytic ehrlichiosis)infected and uninfected mice over the first 4 days of infection. Data from uninfected mice represent combined leukocyte counts from $\mathrm{C} 3 \mathrm{H}, \mathrm{B} 6$, and B6-CD11b $(-/-)$ mice ( $n=10$ mice per time period). Leukocyte counts of infected mice strains are presented separately. All results are presented as the number of cells per $\mu \mathrm{l} \pm \mathrm{SE}$ ( $n=4$ mice per strain per time period). Infected animals were significantly more leukopenic than uninfected controls on Days 3 and 4 after infection.

vidual variation, the total leukocyte numbers were not different between strains of mice. However, B6CD11b (-/-) mice tended towards a more severe leukopenia and neutropenia on Day 3 than either B6 or $\mathrm{C} 3 \mathrm{H}$ mice $(p=0.08)$. In $\mathrm{C} 3 \mathrm{H}$ mice, Ehrlichia morulae were noted in up to $5 \%$ of circulating neutrophils beginning on Day 2 of infection and remained at low levels until Days 5 to 7 after infection. In B6 mice, morulae were rarely noted. This was in stark contrast to B6-CD11b $(-/-)$ mice in which the percent of neutrophils with morulae ranged from $5 \%$ to $26 \%$ at 2 to 6 days after infection. Furthermore, neutrophils of these mice contained large, multiple morulae (Fig. 2).

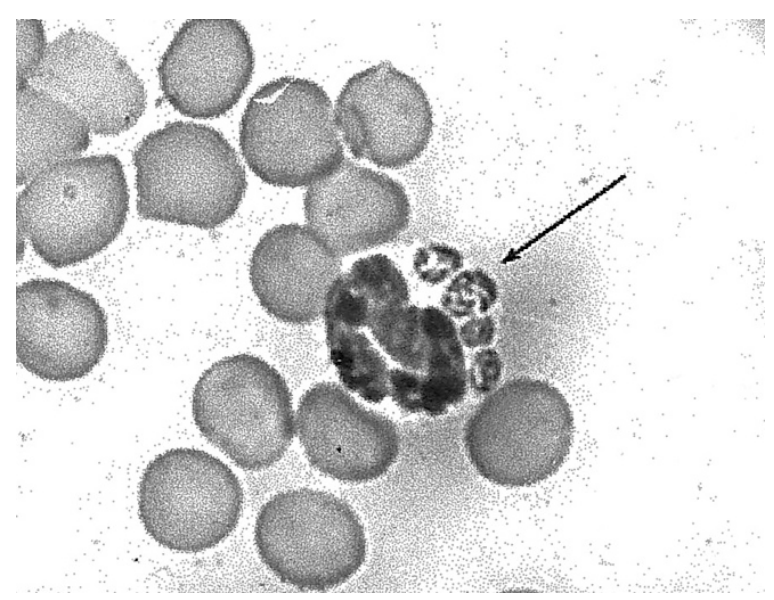

Figure 2.

Peripheral blood smear depicting a neutrophil containing large, multiple, intracytoplasmic morulae from a B6-CD11b (-/-) mouse on Day 2 after infection with the aoHGE. Morula were rarely noted ( $<1$ per 100 neutrophils) in congenic $\mathrm{B} 6$ mice. 
The $\beta 2$ Integrin, CD11b/CD18, Is Up-Regulated on Neutrophils During aoHGE Infection in $\mathrm{C} 3 \mathrm{H}, \mathrm{B} 6$, and B6-IFN $\gamma(-/-)$ Mice

From Days 2 to 4 after infection, there was significant up-regulation of surface CD11b/CD18 in neutrophils from both $\mathrm{C} 3 \mathrm{H}$ and $\mathrm{B} 6$ mice. CD11b/CD18 was upregulated from 2 - to 3 -fold, resulting in an increased percentage of high CD11b/CD18 expressing neutrophils in circulation (Fig. 3, A and B). The population of neutrophils that showed increased CD11b/CD18 expression increased during infection from a baseline of $3 \%$ to $8 \%$ to $18 \%$ to $40 \%$ (Fig. $3 \mathrm{~B}$, upper right quadrant of dot plot). CD11b/CD18 expression remained significantly elevated on a population of neutrophils from infected $\mathrm{C} 3 \mathrm{H}$ and $\mathrm{B} 6$ mice on Days 2, 3, and 4 after infection compared with controls $(p=$ $0.002,0.014$, and 0.003 , respectively). Activation was not evident on Day 6 after infection (Fig. 4A). Neutrophils from infected B6-IFN $\gamma(-/-)$ mice showed significant CD11b/CD18 up-regulation on Days 4 and 6 after infection compared with wildtype uninfected
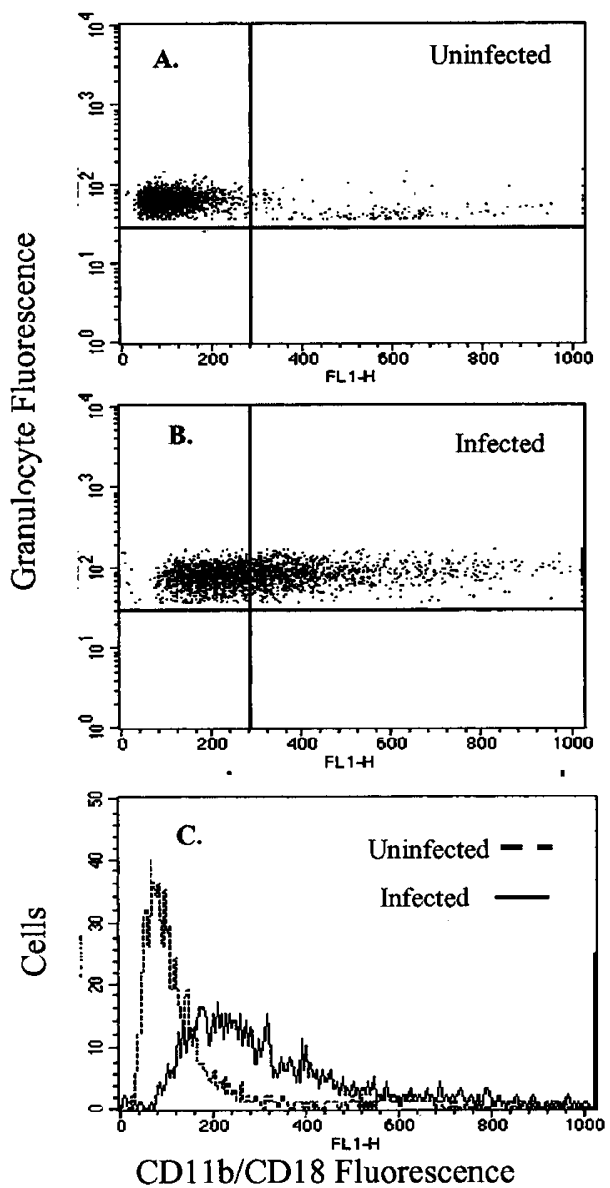

Figure 3.

Flow cytometric analysis of surface expression of the $\beta 2$ integrin CD11b/CD18 on neutrophils during infection with the aoHGE. Representative fluorescence dot plots of CD11b/CD18 on neutrophils from A, uninfected C3H mice on Day 2 after infection; B, infected $\mathrm{C} 3 \mathrm{H}$ mice on Day 2 after infection. There are an increased number of neutrophils (upper right quadrant) that express high CD11b/CD18 fluorescence. C, Overlay fluorescence histogram depicting typical CD11b/CD18 fluorescence from uninfected and infected C3H mice on Day 2 after infection.
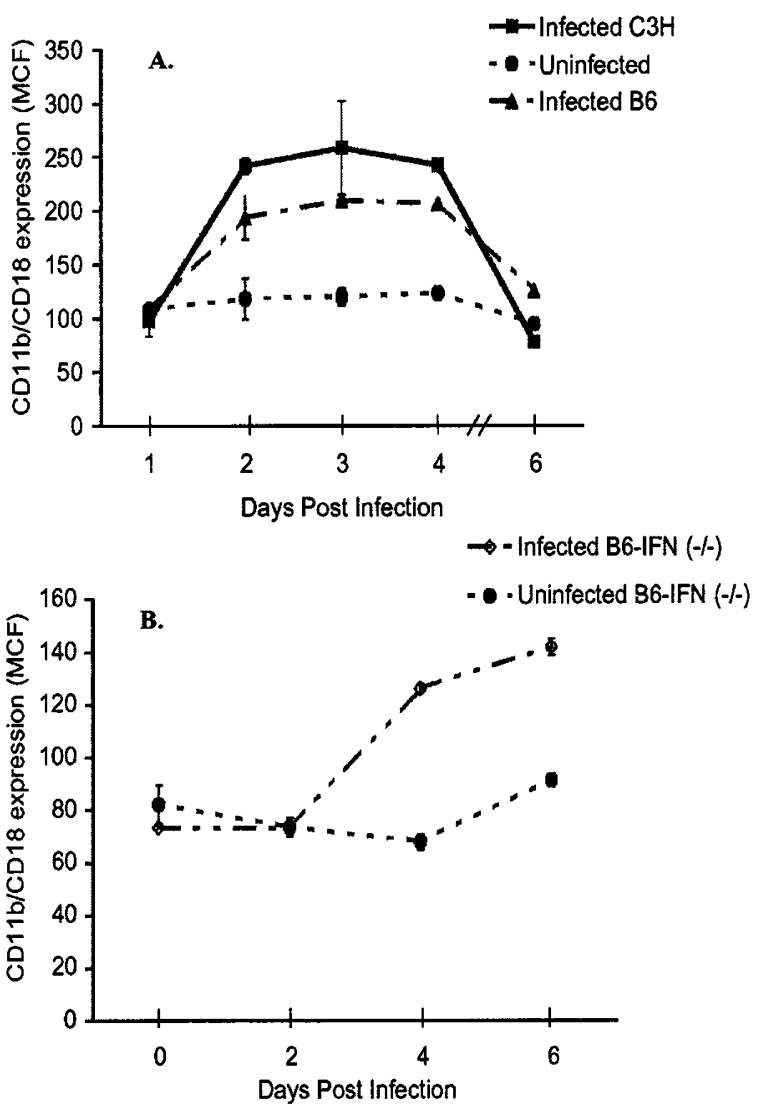

Figure 4.

Mean \pm SE neutrophil CD11b/CD18 fluorescence in infected versus uninfected mice over the first 6 days of infection with the aoHGE ( $n=3$ to 5 mice per group per day). A, Mean channel fluorescence of infected $\mathrm{C} 3 \mathrm{H}$ and $\mathrm{B} 6$ mice compared with uninfected controls. Neutrophils from $\mathrm{C} 3 \mathrm{H}$ and $\mathrm{B} 6$ mice showed a significant up-regulation of CD11b/CD18 on Days 2, 3, and 4 after infection. B, Mean channel fluorescence of infected versus uninfected B6- IFN $\gamma$ $(-/-)$ mice. Neutrophils from infected mice showed significant up-regulation of CD11b/CD18 on Days 4 and 6 after infection.

controls $(p=0.0001, p=0.0005$, respectively; Fig. 4B). Baseline CD11b/CD18 expression on neutrophils from both infected and uninfected wildtype and knock-out mice was lower than in previous experiments. This was attributed to normal experimental or technical variability rather than a true biologic phenomenon. However, the kinetics and extent of neutrophil activation in B6-IFN $\gamma(-/-)$ mice were different than in $\mathrm{C} 3 \mathrm{H}$ and $\mathrm{B} 6$ mice. Initial activation was delayed by at least 24 hours compared with $\mathrm{B} 6$ and $\mathrm{C} 3 \mathrm{H}$ mice, and activation continued to be seen at Day 6 after infection.

\section{CD11b/CD18 Up-Regulation Is Associated with the Presence of Bacteria}

Morphologic evaluation of cytofuge smears was performed on neutrophils separated on the basis of CD11b/CD18 expression. Greater than $97 \%$ of the GR-1-positive events that showed high CD11b/CD18 expression were neutrophils with only rare monocytes noted. Between $6 \%$ and $10 \%$ of these neutrophils were visibly infected, as evidenced by cytoplasmic 
morulae. Of the GR-1-positive events that showed low CD11b/CD18 expression, less than $1.3 \%$ of these neutrophils were visibly infected. These findings suggest that CD11b/CD18 up-regulation is specifically associated with the presence of intracellular bacteria.

\section{AoHGE Infection Kinetics in Blood During Infection of Multiple Strains of Mice}

aoHGE $p 44$ DNA copy number in blood during the first 4 days of infection in $\mathrm{B} 6, \mathrm{C} 3 \mathrm{H}$, and $\mathrm{B} 6-\mathrm{CD} 11 \mathrm{~b}(-/-)$ mice is depicted in Figure 5A. By Day 2, aoHGE DNA copy number significantly increased in the blood from all strains, but B6-CD11b (-/-) had 5-fold higher copy number compared with congenic B6 mice, paralleling the burden noted in the more susceptible $\mathrm{C} 3 \mathrm{H}$ mice (Fig. 5A). At Day 3 after infection, the copy number in the blood decreased in both $\mathrm{C} 3 \mathrm{H}$ and B6-CD11b (-/-) mice. The relative decrease in B6CD11b (-/-) mice was dramatic, likely reflecting their marked neutropenia and bacterial clearance at Day 3. The aoHGE copy number in the blood continued to decrease at Day 4 in $\mathrm{C} 3 \mathrm{H}$ and $\mathrm{B} 6$ mice. However, in the B6-CD11b (-/-) mice, there was an increase

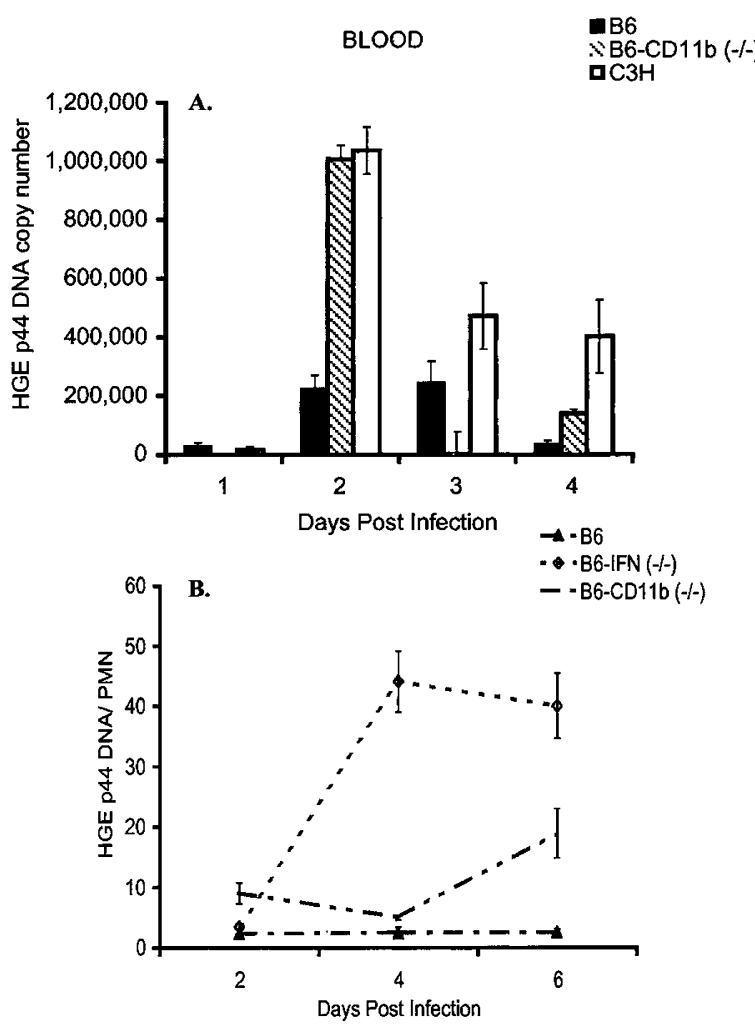

Figure 5.

A, AoHGE $p 44$ DNA copy number in whole blood of infected $\mathrm{C} 3 \mathrm{H}, \mathrm{B} 6$, and B6-CD11b (-l-) mice over the first 4 days of infection. Bars depict mean \pm SE aOHGE $p 44$ DNA copy number per milliliter of blood ( $n=3$ to 5 mice per strain per sample day). B, Ratio of aoHGE p44 DNA copy number per neutrophil in blood of wildtype B6 mice compared with B6-CD11b (-/-) and B6-IFN $\gamma(-/-)$ mice on Days 2, 4 , and 6 after infection. B6-CD11b $(-/-)$ show an increase on Days 2 and 6 after infection compared with wildtype mice, however B6-IFN $\gamma(-/-)$ mice show a greater degree of rickettsemia than both wildtype and B6-CD11b (-/-) mice on Days 4 and 6 after infection, paralleling infection burden. compared with Day 3, probably reflecting both a rebound in neutrophil number as well as bacterial survival. To compare infection burden between experiments and mouse strains, aoHGE $p 44$ copy number in the blood of each mouse was normalized by the number of granulocytes per milliliter of blood. B6-IFN $\gamma$ $(-/-)$ mice showed a marked bacteremia at Days 4 and 6 after infection as evidenced by an increase in aoHGE p44 DNA copy number per granulocyte in blood (Fig. 5B). The level of bacteremia was far greater than noted in wildtype or B6-CD11b $(-/-)$ mice, except at Day 2 when B6-CD11b (-/-) mice showed the highest burden in blood. Interestingly, the increased bacteremia in $\mathrm{B} 6-\mathrm{IFN} \gamma(-/-)$ mice paralleled the kinetics of CD11b/CD18 up-regulation in their blood neutrophils.

\section{AoHGE Replicated More Successfully in Neutrophils Cultured from Infected B6-CD11b(-/-) Mice Than in Neutrophils Cultured from Infected Wild-Type Mice Ex Vivo}

At 36 hours after infection, there was no significant difference in aoHGE copy number in pooled blood from infected B6-CD11b (-/-) and B6 wildtype mice $(1,952,000$ and $2,080,000$ copies of aoHGE p44 DNA per $200 \mu \mathrm{l}$ of blood, respectively). At 48 hours after infection, pooled blood from infected B6-CD11b (-/-) mice had a 4-fold higher aoHGE copy number than B6 wildtype controls $(8,560,000$ and 2,160,000 aoHGE p44 DNA copies per $200 \mu \mathrm{l}$, respectively). AoHGE amplification in cultured neutrophils over time is depicted in Figure 6 . The aoHGE successfully replicated in cultured neutrophils from both strains of mice. Neutrophils isolated after 36 hours of in vivo infection showed a steady increase in aoHGE copy number over the 36-hour culture period with an increase in amplification efficiency at 24 and 32 hours in neutrophils cultured from B6-CD11b (-/-) mice. Neutrophils isolated after 48 hours of in vivo infection from B6CD11b (-/-) mice had twice as much aoHGE p44
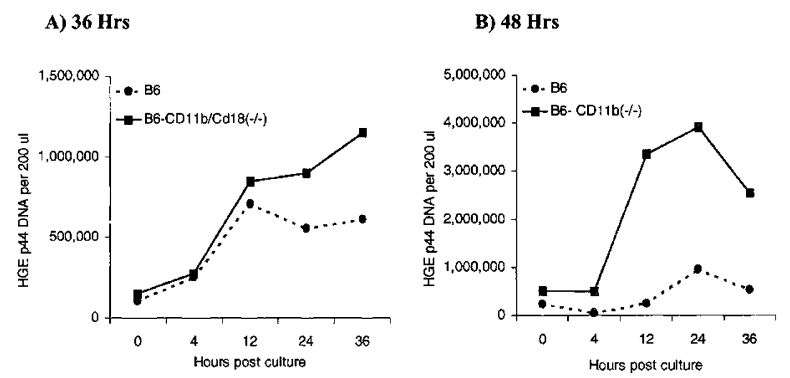

Figure 6.

Quantitative analysis of aoHGE $p 44$ copy number over time in neutrophils harvested and cultured ex vivo from B6 and B6-CD11b (-/-) mice after (A) 36 hours and (B) 48 hours of in vivo infection. Neutrophils harvested from blood after 36 hours of in vivo infection showed no difference between strains, however neutrophils harvested after 48 hours of infection showed a higher initial infection burden in B6-CD11b $(-/-)$ mice. The aoHGE replicated more effectively in neutrophils lacking CD11b/CD18 at 24 and 36 hours after culture despite similar initial infection burden. When initial infection burden was higher, notable differences between cultures were noted as early as 12 hours after culture. 
DNA in their neutrophils than the B6 controls. However, in culture, aoHGE replicated with remarkable success in neutrophils from B6-CD11b (-/-) mice, reaching 4-fold greater aoHGE copy number at 12 to 36 hours after culture compared with B6 wildtype mice. Cultures of neutrophils from uninfected mice were negative for aoHGE DNA at all time points sampled.

\section{Discussion}

The aoHGE is an obligate intracellular bacterium reliant upon its host granulocyte for systemic spread of infection, immune evasion, and transmission to a new vector. As such, the aoHGE is likely to affect its host cell in a number of ways. It was hypothesized that infection of neutrophils with the aoHGE may result in leukocyte activation and changes in receptor expression that determine some features of disease, such as leukopenia, bacterial survival, and cell clearance. We demonstrated that infection of multiple strains of mice with the aoHGE resulted in activation of neutrophils, as evidenced by up-regulation of the integrin CD11b/ CD18 to the surface of the cell. This activation was directly associated with the presence of intracellular bacteria, occurred in the absence of IFN- $\gamma$ production, and seemed to be associated with early effective cellular clearance of the organism, because infection of B6 mice lacking CD11b/CD18 resulted in an initial increase in bacterial burden. Although the mechanism(s) responsible for the increased susceptibility of B6-CD11b (-/-) mice to HGE infection was not fully elucidated, in vitro experiments suggested that defects in bacterial killing associated with exponential bacterial replication may be partially responsible. Although CD11b/CD18 plays a role in leukocyte adhesion to endothelium, the absence of this integrin did not alter the time frame or degree of peripheral leukopenia.

The $\beta_{2}$ integrin, CD11b/CD18, is found both on the surface of neutrophils as well as intracellularly within specific cytoplasmic granules. Numerous signals, including cytokines such as IFN- $\gamma$, chemotactic factors, ligation, and cross-linking of L-selectin or P-selectin glycoprotein ligand-1 (PSGL-1) result in increased $\beta_{2}$ integrin surface expression and activation (Blanks et al, 1998; Simon et al, 1995). Engagement of CD11b/ CD18 promotes phagocytosis of complementopsonized bacteria, cell spreading, cell adhesion to the microvasculature, extravasation into tissues, chemotaxis, and intracellular signaling with resultant increases in intracellular calcium, gene expression, and oxygen consumption associated with the oxidative burst (Berton et al, 1996; Dib, 2000). Accordingly, studies on neutrophil function in mice lacking CD11b/ CD18 show a decreased ability to phagocytize opsonized bacteria, generate an oxidative burst, or undergo homotypic aggregation (Coxon et al, 1996; Lu et al, 1997).

Intraperitoneal inoculation of the aoHGE was an effective means of infection in CD11b (-/-) knock-out mice. We chose this method of infection because previous studies in our laboratory established that challenge by either tick bite or intraperitoneal injection are equally effective for infecting laboratory mice with the aoHGE and result in similar infection kinetics (Hodzic et al, 2001). In addition, most research suggests that the $\beta 2$ integrins play a limited role in neutrophil migration to the peritoneum and thus to the site of inoculation. Data on neutrophils from CD18, CD11a/CD18, and CD11b/CD18 knock-out mice all show that extravasation into the peritoneum is more or less intact in response to diverse stimuli including thioglycollate and Streptococcus pneumoniae (Coxon et al, 1996; Lu et al, 1997; Mizgerd et al, 1997). This may be a result of the redundancy in adhesion molecule participation in inflammatory cell trafficking in knock-out mice or alternative pathways for neutrophil emigration during peritonitis (such as $\beta 1$ integrinvascular cell adhesion molecule-1 interaction). Mice lacking CD11a/CD18 also become efficiently infected via intraperitoneal injection of aoHGE (our unpublished observations).

We hypothesized that leukopenia during acute aoHGE infection may reflect altered leukocyte distribution with increased endothelial sequestration or margination mediated, in part, by CD11b/CD18. The current study clearly demonstrated that peripheral leukopenia develops in multiple strains of mice and in this way parallels natural disease. However, leukopenia develops independent of infection burden and occurs in the same time frame and to the same degree in mice lacking CD11b/CD18 as in other infected mice. This suggests that either neutrophil emigration/margination is not a mechanism of leukopenia during infection or that other adhesion molecules are involved. In support of this, others have demonstrated a dominant role for CD11a/CD18 in effective neutrophil emigration (Lu et al, 1997). It is interesting to note that the leukopenia in blood corresponded temporally with bacterial clearance from the blood.

We examined whether neutrophil activation was a generalized systemic response to infection or whether it was related to direct neutrophil-pathogen association. Morphologic evaluation of neutrophils sorted by FACS on the basis of high versus low CD11b/CD18 expression, showed that high CD11b/CD18 expression was linked to high infection burden. Thus, bacterial attachment, endocytosis, or replication likely result in CD11b/CD18 up-regulation. Activation may result in clearance of the infected neutrophil. This is suggested by the fact that absence of CD11b/CD18 is permissive to an initial increase in bacteremia. Furthermore, the time course of infection and neutrophil activation parallel each other such that as infection burden decreases, so does the number of activated neutrophils in circulation. Others have demonstrated that the aoHGE elicits alterations in its host cell simply via bacterial contact with its host cell receptor and/or internalization rather than via bacterial protein synthesis and proliferation (Mott and Rikihisa, 2000; Yoshiie et al, 2000). This may explain why mouse strains with very different infection kinetics and infection burden show both neutrophil activation and leukopenia. 
Given the importance of IFN- $\gamma$ in the murine cytokine response to aoHGE and its potential pleiotropic functions, we evaluated its role in neutrophil activation. We found that neutrophils from B6-IFN $\gamma(-/-)$ mice showed significant up-regulation of CD11b/ CD18 in response to infection. However, neutrophil activation was delayed in onset, less pronounced, and persisted longer than in wildtype mice. This pattern of activation paralleled infection kinetics in blood, with bacterial burden increasing later in infection and persisting longer. Results are consistent with the findings that neutrophil CD11b/CD18 up-regulation is directly associated with the presence of bacteria. One potential direct mechanism by which bacterial contact or infection may result in CD11b/CD18 up-regulation is via PSGL-1. It was recently demonstrated that the aoHGE directly infects human neutrophils via receptor-mediated endocytosis using PSGL-1 as a receptor (Goodman et al, 1999; Herron et al, 2000). As stimulation of PSGL-1 activates mouse neutrophils, it is indeed possible that binding of aoHGE is an initiating event that leads to cellular activation and increased expression of CD11b/CD18 on the host cell surface (Blanks et al, 1998).

Both B6-CD11b (-/-) mice and B6-IFN $\gamma(-/-)$ mice showed increased bacteremia during aoHGE infection compared with wildtype mice. However, the kinetics of infection in these mice differed. By direct comparison of infection burden using a ratio of $\mathrm{HGE}$ DNA p44 copy number per neutrophil, it is clear that IFN- $\gamma$ is more important, ultimately, in limiting bacterial number and persistence than CD11b/CD18. However, it is likely that the extent of CD11b/CD18 activation and its role in aoHGE clearance is partially dependent on IFN- $\gamma$. This is consistent with other reports of the dominant role of IFN- $\gamma$ in controlling HGE infection (Akkoyunlu and Fikrig, 2000; Dumler et al, 2000; Martin et al, 2001).

The defect in B6-CD11b (-/-) mice likely reflects a decreased ability of neutrophils to kill or clear the bacterial agent during the first 48 to 72 hours of infection. Although bacterial attachment and entry were not directly measured, bacteria were clearly able to enter and replicate within neutrophils from B6CD11b (-/-) mice, as evidenced by the increased percentage of circulating neutrophils with large intracytoplasmic morulae compared with wildtype mice. Recent in vitro work with murine splenic neutrophils suggests that the aoHGE is able to repress the respiratory burst of its host cell via down-regulation of gp91 $1^{\text {phox }}$, a component of the bacteriocidal NADPH oxidase system (Banerjee et al, 2000). Similarly, the aoHGE has been shown to inhibit superoxide anion generation by human neutrophils in vitro (Mott and Rikihisa, 2000). Given the diminished oxidative burst shown for neutrophils from B6-CD11b (-/-) mice (Coxon et al, 1996; Lu et al, 1997), these two effects may act synergistically and lead to the aoHGE's survival and proliferation in its host cell. Finally, although $\beta 2$ integrin knock-out mice are not known to have any defects in IFN- $\gamma$ production, we cannot exclude the potential role of decreased proinflamma- tory gene production in CD11b/CD18 mice as a possible mechanism of the increased bacteremia. Our data are compatible with the findings that reduction of cellular activation is beneficial to organism survival and proliferation.

Ex vivo culture of neutrophils from infected B6CD11b (-/-) mice demonstrated that lack of CD11b/ CD18 was permissive to organism replication both when the infection burden was initially the same (36 hours) and when the burden was initially higher (neutrophils harvested at 48 hours after infection). Although viability of neutrophils in culture was not directly measured, Ehrlichia are obligate intracellular pathogens that lose their infectivity within hours after becoming extracellular (Park and Rikihisa, 1991). In addition, the aoHGE has been shown to delay apoptosis of human neutrophils in vitro, with infected cells in culture remaining viable for up to 96 hours (Yoshiie et al, 2000). Thus, the marked increase in aoHGE DNA in neutrophils cultured for 36 hours was consistent with previous data suggesting delayed apoptosis of neutrophils in culture. This experiment reinforces the notion that CD11b/CD18 may play an important, early role in cell signaling and effective bacterial killing or clearance during acute infection with the aoHGE.

In conclusion, CD11b/CD18 up-regulation may occur upon attachment or entry of aoHGE into its host granulocyte. Increased CD11b/CD18 expression seems to be associated with the presence of morulae. Activation in and of itself may lead to bacterial killing or neutrophil clearance if the aoHGE did not possess other mechanisms by which to circumvent the normal microbicidal capacities of its host cell. This then may explain the apparent paradox that the absence of CD11b/CD18, and thus cell activation, leads to a moderate, early increase in bacteremia and yet activated cells are also the highly infected cells. Further studies to elucidate the timing and mechanism of CD11b/CD18 up-regulation are needed to fully define the events leading to cell activation and clearance.

\section{Materials and Methods}

\section{Mice}

Female, 5- to 8-week-old, specific pathogen-free $\mathrm{C} 3 \mathrm{H} / \mathrm{HeN}(\mathrm{C} 3 \mathrm{H})$ and $\mathrm{C} 3 \mathrm{H} / \mathrm{Smn}$.ClcrHsd-scid (SCID) mice were purchased from National Cancer Institute Animal Production Program, Frederick Cancer Research Center (Frederick, Maryland) and Harlan Sprague-Dawley (Indianapolis, Indiana), respectively. C57BL/6J (B6) and B6.129S7-Ifng ${ }^{\text {tm1Ts }}$ [B6-IFN $\gamma(-/$ -)] were purchased from The Jackson Laboratory (Bar Harbor, Maine). Thirty female, 5- to 12- week-old CD11b/CD18 knock-out mice [B6-CD11b (-/-)], backcrossed onto a B6 genotype for 12 generations, were generously provided by Dr. Christie Ballantyne (Baylor College of Medicine, Departments of Medicine and Pediatrics, Houston, Texas). Age and sexmatched wildtype B6 mice were used as congenic controls for both B6-CD11b (-/-) and B6-IFN $\gamma(-/-)$ 
mice. Mice were maintained according to approved institutional animal use and care protocols.

\section{AoHGE and In Vivo Experimental Infection}

The aoHGE used in this study was isolated from peripheral blood of an HGE patient from Nantucket, Massachusetts ( $\mathrm{NCH}-1$ isolate) (Telford et al, 1995). Infection with the aoHGE was maintained through serial intraperitoneal passage of infected blood in SCID mice, as previously described (Hodzic et al, 1998). Blood from these mice served as the source of infectious material in all experiments. For each experiment, infected SCID mouse blood was pooled, and 50 $\mu \mathrm{l}$ was set aside for quantitative PCR to determine the initial infectious dose of aoHGE. Pooled blood was used to help insure approximately equivalent inocula of bacteria per mouse. Experimental mice were inoculated intraperitoneally with $100 \mu \mathrm{l}$ of this pooled infected, anticoagulated blood. Control mice were inoculated intraperitoneally with $100 \mu \mathrm{l}$ of uninfected anticoagulated SCID mouse blood. In these experiments, groups of three to five infected and control mice were killed with $\mathrm{CO}_{2}$ from Days 1 to 6 after infection, depending on experimental design. At necropsy, blood was obtained for quantitative PCR, flow cytometry, white blood cell counts, and blood smear analysis. Experiments involving $\mathrm{C} 3 \mathrm{H}$ and $\mathrm{B} 6$ mice were repeated three times. Because of limited mouse availability, the 4- to 6-day in vivo experiments involving B6-CD11b (-/-) and B6-IFN $\gamma(-/-)$ mice were performed in completion once. Portions of the experiment using B6-CD11b (-/-) mice were repeated both in vivo and ex vivo to confirm the marked increase in infection burden in knock-out mice and the role of CD11b/CD18 in bacterial killing (see below).

\section{Hematology}

Whole blood was collected by cardiocentesis and placed into tubes containing EDTA (Becton Dickinson, Rutherford, New Jersey). Leukocyte counts were performed within 6 hours of blood collection on a Serano Baker 9000 automated cell-counting instrument (Biochemical Immunosystems, Allentown, Pennsylvania). Blood smears were made for each animal and stained with Protocol Hema 3; and leukocyte differential counts were performed on 200 cells.

\section{Flow Cytometric Analysis}

CD11b/CD18 expression on peripheral neutrophils was assessed by flow cytometry of whole blood obtained from infected and uninfected mice. Whole blood was used to minimize sample handling and decrease the risk of ex vivo neutrophil activation. Briefly, $100 \mu \mathrm{l}$ of EDTA anticoagulated whole blood from both infected and uninfected control animals was diluted with $100 \mu \mathrm{l}$ of flow wash buffer (PBS/0.1\% $\mathrm{NaN}_{3} / 1.0 \%$ FCS). Blood was labeled with rat antimouse antibodies to detect both the GR-1 antigen (clone RB6-8C5; PE conjugated; specific for murine granulocytes; PharMingen, San Diego, California) and
CD11b/CD18 (clone Ml/70.15; FITC conjugated; CalTag Laboratories, Burlingame, California). Fifty microliters of GR-1 diluted 1:250 and $10 \mu \mathrm{l}$ of CD11b/ CD18 antibody were added to each reaction. In addition, $1 \mu \mathrm{g} / \mathrm{ml}$ of LDS-751, a vital nucleic acid stain, was used to trigger acquisition of nucleated cells (Exciton Chemical Company Inc., Dayton, Ohio) (Simon et al, 1992). Reactions were incubated for 30 minutes on ice in the dark. After incubation, $200 \mu$ l of flow wash buffer was added along with $300 \mu \mathrm{l}$ of $1 \%$ paraformaldehyde in PBS to fix the cells and bring the final dilution of whole blood to 1:5. For all experiments, samples were run on a FACScan (Becton Dickinson Immunocytometry Systems, San Jose, California) flow cytometer. Events were gated on LDS-751-positive cells (FL3 channel), and CD11b/CD18 expression was evaluated on GR-1-positive cells (granulocytes). Parameters were stored in list mode and analyzed using FACScan research software (Becton Dickinson Immunocytometry Systems).

\section{Cell Sorting}

Nine $\mathrm{C} 3 \mathrm{H} / \mathrm{HeN}$ mice were infected as described above. On Days 2, 3, and 4 after infection, whole blood from three infected mice and one uninfected mouse was stained with GR-1, LDS-751, and CD11b/CD18 as above. Blood (400 to $500 \mu \mathrm{l}$ ) from each mouse was sorted on a MoFlo Cell Sorter (Cytomation, Fort Collins, Colorado). The threshold was set on LDS-751 positive cells (nucleated cells), and a two-way sort was performed to separate and collect GR-1-positive events (neutrophils) that expressed high CD11b/CD18 fluorescence versus those that expressed low CD11b/ CD18 fluorescence. Cells were collected into PBS + $5 \%$ FCS on ice. The total number of events collected was tallied. A cytofuge smear was prepared from a $100-\mu \mathrm{l}$ aliquot of each sorted cell population from each mouse (Cytospin2; Shandon Lipshaw, Pittsburgh, Pennsylvania). Slides were stained with a modified Wrights Giemsa stain. A 300 cell differential count was performed to assess cell purity and calculate the percent of infected neutrophils in high versus low CD11b/CD18 expressors.

\section{DNA Extraction and Quantitative PCR}

DNA Extraction. DNA was extracted from $50 \mu \mathrm{l}$ of blood or $200 \mu \mathrm{l}$ of cultured or sorted neutrophils. Extraction of genomic DNA was performed using the QIAGEN DNeasy Tissue Kit (QIAGEN, Valencia, California) according to the manufacturer's instruction for animal blood and tissues.

TaqMan Probe and Primers Design. Three oligonucleotides, two primers, and one probe for the target gene HGE p44 (GenBank accession no. AF037599) were selected using Primer Express software (PerkinElmer, Applied Biosystems, Foster City, California). Primers P44-802F (5'-ACGTCGAAAAAGGCGGAAA$\left.3^{\prime}\right)$ and P44-872R (5'-GCTACAGCTGCCGCGTTATC$\left.3^{\prime}\right)$ were synthesized to amplify a 71-bp fragment of a C-terminal conserved region of the HGE p44 gene. 
The internal oligonucleotide probe P44-825P (5'TGCAGGCACTCCGGAACCCGT-3') was labeled at the $5^{\prime}$ end with the reporter dye FAM (6-carboxyfluorescein) and at the $3^{\prime}$ end with the quencher dye TAMRA (6-carboxy-tetramethyl-rhodamine).

TaqMan PCR Reaction Mix and Thermal Cycling. The 25- $\mu$ l PCR mixture contained $10 \mu$ l of diluted template or plasmid standard, $400 \mathrm{~nm}$ of each primer, $80 \mathrm{~nm}$ fluorogenic probe, and mastermix (TaqMan Universal PCR Master Mix; Perkin-Elmer, Applied Biosystems). Mastermix contained $0.25 \mathrm{U}$ of AmpErase UNG, $1.6 \mathrm{~mm}$ deoxynucleoside triphosphates dNTP, $0.625 \mathrm{U}$ of AmpliTaq Gold, $60 \mathrm{~mm} \mathrm{KCl,} 12 \mathrm{~mm}$ Tris- $\mathrm{HCl}$ (pH 8.3), $12 \mathrm{~mm}$ EDTA, and $7.5 \mathrm{~mm} \mathrm{MgCl}_{2}$. DNA amplification, data acquisition, and data analysis were performed in an ABI Prism 7700 Sequence Detector (Perkin-Elmer, Applied Biosystems). The PCR thermal cycling parameters started with two initial steps: 2 minutes at $50^{\circ} \mathrm{C}$ and denaturation for 10 minutes at $95^{\circ} \mathrm{C}$; this was followed by 40 cycles of two steps: 15 seconds of denaturation at $95^{\circ} \mathrm{C}$ and 1 minute of annealing at $60^{\circ} \mathrm{C}$. Quantification of the amount of HGE p44 gene in each sample was accomplished by measuring CT and using an absolute standard curve to determine starting copy number. To quantify copy number of HGE p44 gene in the samples, a plasmid standard was prepared as previously described (Hodzic et al, 2001). A Sequence Detection System (version 1.6 software) was used to analyze data.

\section{Ex Vivo Culture of Mouse Neutrophils}

Differences in the ability of aoHGE to successfully replicate in neutrophils from infected B6 and B6CD11b (-/-) mice was investigated ex vivo. In brief, 12 mice were infected ( 6 knockouts, 6 wild type) and 8 mice served as uninfected controls (4 knockouts, 4 wild type) as described above. Blood was obtained from three infected and two uninfected mice per group via cardiocentesis at 36 and 48 hours after intraperitoneal inoculation. These time periods were selected because at 36 hours there is no difference in aoHGE p44 DNA copy number in blood between wild type and knock-out mice, whereas at 48 hours there is a significant difference with a marked increase in knockout mice. Blood from each group was pooled with one infected and one uninfected group of B6 and B6CD11b (-/-) per time period represented. Fifty microliters of pooled blood per group was frozen for PCR to quantitate infection burden at 36 and 48 hours. Neutrophils were isolated from whole blood using a density gradient neutrophil isolation media according to the manufacturer's instructions (NIM-2; Cardinal Associates Inc., Santa Fe, New Mexico). After washing, neutrophils were resuspended in RPMI-1640 media supplemented with 10\% FCS (GIBCO, Grand Island, New York) to a final concentration of approximately 5 $\times 10^{5} \mathrm{cells} / \mathrm{ml}$ and plated in a 24-well, flat-bottomed tissue culture plate (Linbro; ICN Biomedicals, Aurora, Ohio). Neutrophil viability, as determined by trypan blue exclusion, was determined to be greater than $95 \%$. Neutrophil purity was assessed by cytofuge preparation and Protocol Hema 3 staining (modified Diffquick stain; Baxter, Miami, Florida) and determined to be between $75 \%$ and $94 \%$ pure. Culture plates were maintained in a $37^{\circ}$ incubator with $5 \% \mathrm{CO}_{2}$. Neutrophils were sampled at the time of culture (0 hours) and at 4, 12, 24, and 36 hours after culturing. For each sample period, $200 \mu$ l of cultured neutrophils was removed and frozen. Later, DNA was extracted, and quantitative PCR for aoHGE was performed (as above).

\section{Statistics}

Statistical comparisons between infected and uninfected mice were made using a Student's $t$ test (SPSS, version 6.1 for Windows ${ }^{\mathrm{TM}}$; SPSS Inc., Chicago, Illinois). Multiple comparison analysis was made using a one-way ANOVA, followed by a least squares difference post-hoc test. Calculated $p$ values $<0.05$ were considered significant.

\section{Acknowledgements}

We thank Ms. Kim Freet, Ms. Amy Smith, Dr. Sunlian Feng, Ms. Carol Oxford, Dr. David Pearson, Mr. Aaron Lum, and Ms. Betsy Priest for valuable assistance and technical support.

\section{References}

Aas V, Larsen K, and Iversen JG (1999). Interferon-gamma elicits a G-protein-dependent $\mathrm{Ca}_{2}{ }^{+}$signal in human neutrophils after depletion of intracellular $\mathrm{Ca}_{2}{ }^{+}$stores. Cell Signal 11:101-110.

Akkoyunlu M and Fikrig E (2000). Gamma interferon dominates the murine cytokine response to the agent of human granulocytic ehrlichiosis and helps to control the degree of early rickettsemia. Infect Immun 68:1827-1833.

Anderson BE, Dawson JE, Jones DC, and Wilson KH (1991). Ehrlichia chaffeensis, a new species associated with human ehrlichiosis. J Clin Microbiol 29:2838-2842.

Banerjee R, Anguita J, Roos D, and Fikrig E (2000). Cutting edge: Infection by the agent of human granulocytic ehrlichiosis prevents the respiratory burst by down-regulating gp91phox. J Immunol 164:3946-3949.

Berton G, Yan SR, Fumagalli L, and Lowell CA (1996). Neutrophil activation by adhesion: Mechanisms and pathophysiological implications. Int J Clin Lab Res 26:160-177.

Blanks JE, Moll T, Eytner R, and Vestweber D (1998). Stimulation of P-selectin glycoprotein ligand-1 on mouse neutrophils activates beta 2 -integrin mediated cell attachment to ICAM-1. Eur J Immunol 28:433-443.

Chen SM, Dumler JS, Bakken JS, and Walker DH (1994). Identification of a granulocytotropic Ehrlichia species as the etiologic agent of human disease. J Clin Microbiol 32:589595.

Coxon A, Rieu P, Barkalow FJ, Askari S, Sharpe AH, von Andrian UH, Arnaout MA, and Mayadas TN (1996). A novel role for the beta 2 integrin CD11b/CD18 in neutrophil apoptosis: A homeostatic mechanism in inflammation. Immunity 5:653-666. 
Dib K (2000). BETA 2 integrin signaling in leukocytes. Front Biosci 5:D438-D451.

Dumler JS, Trigiani ER, Bakken JS, Aguero-Rosenfeld ME, and Wormser GP (2000). Serum cytokine responses during acute human granulocytic ehrlichiosis. Clin Diagn Lab Immunol 7:6-8.

Gaviria JM, van Burik JA, Dale DC, Root RK, and Liles WC (1999). Comparison of interferon-gamma, granulocyte colony-stimulating factor, and granulocyte-macrophage colony-stimulating factor for priming leukocyte-mediated hyphal damage of opportunistic fungal pathogens. J Infect Dis 179:1038-1041.

Goodman JL, Nelson CM, Klein MB, Hayes SF, and Weston BW (1999). Leukocyte infection by the granulocytic ehrlichiosis agent is linked to expression of a selectin ligand. $\mathrm{J}$ Clin Invest 103:407-412.

Herron MJ, Nelson CM, Larson J, Snapp KR, Kansas GS, and Goodman JL (2000). Intracellular parasitism by the human granulocytic ehrlichiosis bacterium through the P-selectin ligand, PSGL-1. Science 288:1653-1656.

Hodzic E, Feng S, Fish D, Leutenegger CM, Freet KJ, and Barthold SW (2001). Infection of mice with the agent of human granulocytic ehrlichiosis after different routes of inoculation. J Infect Dis 183:1781-1786.

Hodzic E, ljdo JW, Feng S, Katavolos P, Sun W, Maretzki CH, Fish D, Fikrig E, Telford SR 3rd, and Barthold SW (1998). Granulocytic ehrlichiosis in the laboratory mouse. J Infect Dis 177:737-745.

Kullberg BJ, van't Wout JW, Hoogstraten C, and van Furth R (1993). Recombinant interferon-gamma enhances resistance to acute disseminated Candida albicans infection in mice. $\mathrm{J}$ Infect Dis 168:436-443.

Lu H, Smith CW, Perrard J, Bullard D, Tang L, Shappell SB, Entman ML, Beaudet AL, and Ballantyne CM (1997). LFA-1 is sufficient in mediating neutrophil emigration in Mac-1deficient mice. J Clin Invest 99:1340-1350.
Martin ME, Caspersen K, and Dumler JS (2001). Immunopathology and ehrlichial propagation are regulated by interferon-gamma and interleukin-10 in a murine model of human granulocytic ehrlichiosis. Am J Pathol 158:18811888.

Mizgerd JP, Kubo H, Kutkoski GJ, Bhagwan SD, Scharffetter-Kochanek K, Beaudet AL, and Doerschuk CM (1997). Neutrophil emigration in the skin, lungs, and peritoneum: Different requirements for CD11/CD18 revealed by CD18-deficient mice. J Exp Med 186:1357-1364.

Mott J and Rikihisa Y (2000). Human granulocytic ehrlichiosis agent inhibits superoxide anion generation by human neutrophils. Infect Immun 68:6697-6703.

Park J and Rikihisa Y (1991). Inhibition of Ehrlichia risticii infection in murine peritoneal macrophages by gamma interferon, a calcium ionophore, and concanavalin A. Infect Immun 59:3418-3423.

Simon SI, Burns AR, Taylor AD, Gopalan PK, Lynam EB, Sklar LA, and Smith CW (1995). L-selectin (CD62L) crosslinking signals neutrophil adhesive functions via the Mac-1 (CD11b/CD18) beta 2-integrin. J Immunol 155:1502-1514.

Simon SI, Chambers JD, Butcher E, and Sklar LA (1992). Neutrophil aggregation is beta 2-integrin- and L-selectindependent in blood and isolated cells. J Immunol 149:27652771.

Telford SR 3rd, Lepore TJ, Snow P, Warner CK, and Dawson JE (1995). Human granulocytic ehrlichiosis in Massachusetts. Ann Intern Med 123:277-279.

Walker DH and Dumler JS (1996). Emergence of the ehrlichioses as human health problems. Emerg Infect Dis 2:1829.

Yoshiie K, Kim HY, Mott J, and Rikihisa Y (2000). Intracellular infection by the human granulocytic ehrlichiosis agent inhibits human neutrophil apoptosis. Infect Immun 68:1125-1133. 